\title{
Single Molecule Investigation of Nanoconfinement Hydrophobicity in Heterogeneous Catalysis
}

Bin Dong, ${ }^{\dagger, \S}$ Nourhan Mansour, ${ }^{\dagger, \S}$ Yuchen Pei, ${ }^{\ddagger \S}$ Zhuoran Wang, ${ }^{\ddagger}$ Tengxiang Huang, ${ }^{\dagger}$ Seth L. Filbrun, ${ }^{\dagger}$ Minda Chen,${ }^{\ddagger}$ Xiaodong Cheng, ${ }^{\dagger}$ Marek Pruski, ${ }^{\ddagger}$ Wenyu Huang, $,{ }^{\dagger},{ }^{*}$ Ning Fang ${ }^{\dagger, *}$

†Department of Chemistry, Georgia State University, Atlanta, Georgia 30303, United States

\#Department of Chemistry, Iowa State University, and Ames Laboratory, U.S. Department of Energy, Ames, Iowa 50011, United States

$\S$ These authors contributed equally to this work.

*To whom correspondence should be addressed. E-Mail: nfang@gsu.edu and whuang@iastate.edu. 


\section{Table of Contents}

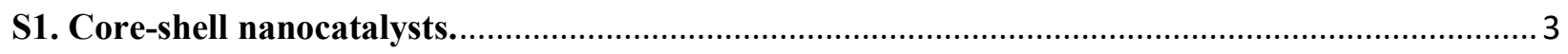

S1.1. Synthesis of bare core-shell nanocatalysts .............................................................................. 3

S1.2. Synthesis of $\mathrm{CF}_{3}$-functionalized core-shell nanocatalysts by post-synthesis method...................... 4

S1.3. Synthesis of $\mathrm{SO}_{3} \mathrm{H}$-functionalized core-shell nanocatalysts by post-synthesis method ................... 4

S1.4. Quantification of $\mathrm{CF}_{3}$ - and $\mathrm{SO}_{3} \mathrm{H}$-functional group in core-shell nanocatalysts ......................... 7

S1.5. Reagents used in the synthesis of nanocatalysts ...................................................................... 9

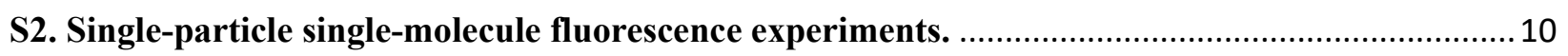

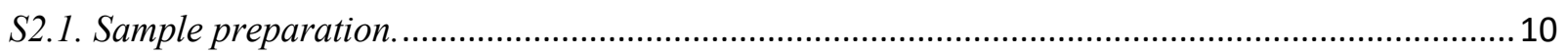

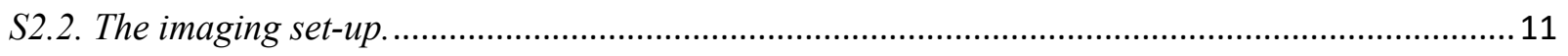

S2.3. Quantify reaction dynamics at single particle single-molecule level with turn-over resolution. .. 13

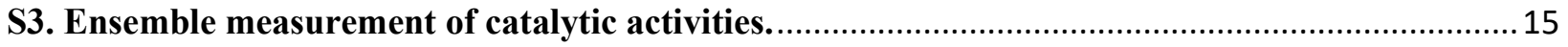

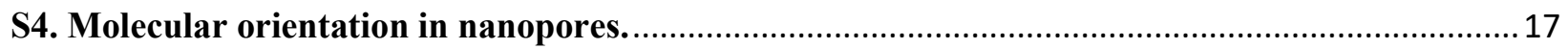

S5. Reaction mechanisms of the oxidation of Amplex red. ......................................................... 18

S6. Ensemble measurement of activation energy of catalytic reaction in nanopores. ....................... 19

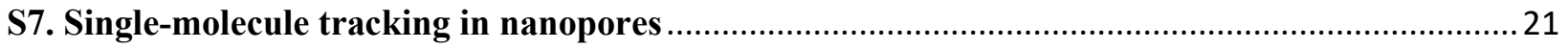

S7.1. Super-localization of the center positions of single-molecule images. .........................................2 21

S7.2. Mean squared displacement analysis of single-molecule transport in nanopores. .......................22

S8. Enrichment of resorufin molecules in nanopores. .................................................................. 25

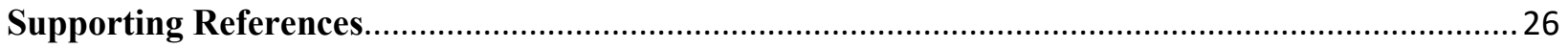




\section{S1. Core-shell nanocatalysts.}

S1.1. Synthesis of bare core-shell nanocatalysts

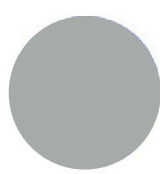

$\mathrm{SiO}_{2}$

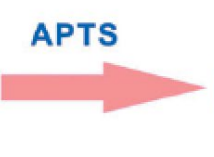

$\mathrm{NH}_{2}-\mathrm{SiO}_{2}$
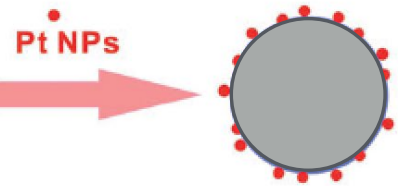

TEOS/C $\mathrm{C}_{16} \mathrm{TAB}$

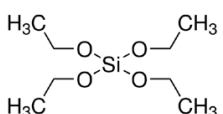

$\mathrm{Pt} / \mathrm{NH}_{2}-\mathrm{SiO}_{2}$

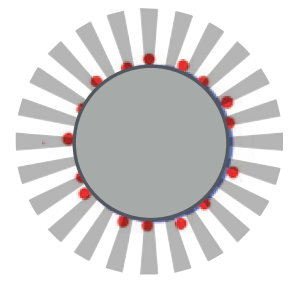

Figure S1. Schematic synthesis strategy for the core-shell nanocatalysts.

Synthesis of bare core-shell nanocatalysts with $100 \mathrm{~nm} \mathrm{SiO} 2$ core, $5 \mathrm{~nm}$ Pt nanoparticles, and 120 $\mathrm{nm}$ mesoporous silica ( $\sim 3 \mathrm{~nm}$ pore) are described in detail in our previous work. ${ }^{l}$ Figure $\mathrm{S} 1$ is a schematic synthesis strategy for this core-shell nanocatalysts. Briefly, we first prepared uniform $100 \mathrm{~nm} \mathrm{SiO} 2$ cores with seeded growth method, and then functionalized its surface with $\mathrm{NH}_{2-}$
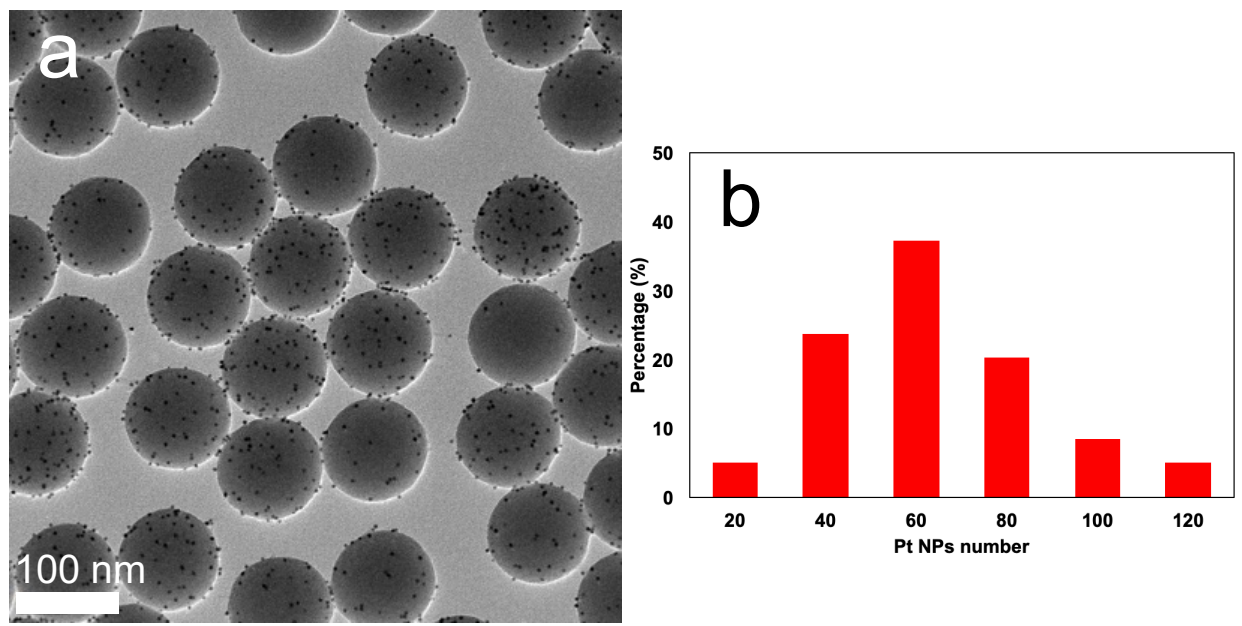

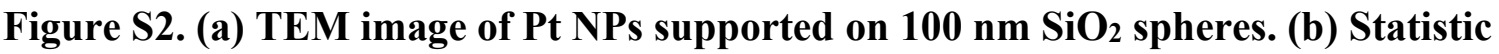
numbers of Pt NPs on $\mathrm{SiO}_{2}$ spheres.

groups to prepare $\mathrm{NH}_{2}-\mathrm{SiO}_{2}$ by the hydrolysis of (3-Aminopropyl) triethoxysilane (APTS). Previously prepared $5 \mathrm{~nm} \mathrm{Pt}$ nanoparticles (active sites) were anchors on $\mathrm{NH}_{2}-\mathrm{SiO}_{2}$ by impregnation method to prepare $\mathrm{Pt} / \mathrm{NH}_{2}-\mathrm{SiO}_{2}$. There are round $56 \pm 23 \mathrm{Pt}$ particles per $\mathrm{SiO}_{2}$ sphere 
(Figure S2). Though Pt NPs are distributed well on the $\mathrm{SiO}_{2}$ surface, the particle-to-particle deviation is not small. The mesoporous silica shells were finally coated on $\mathrm{Pt} / \mathrm{NH}_{2}-\mathrm{SiO}_{2}$ by the controlled hydrolysis of tetraethyl orthosilicate (TEOS) in a bilayer solution (water-ammoniaethanol layer and hexane layer) with hexadecyltrimethylammonium bromide $\left(\mathrm{C}_{16} \mathrm{TAB}\right)$ as the surfactant.

\section{S1.2. Synthesis of $\mathrm{CF}_{3}$-functionalized core-shell nanocatalysts by post-synthesis method}

In a typical synthesis, $100 \mathrm{mg}$ bare core-shell nanocatalysts with a $3 \mathrm{~nm}$ pore size were dispersed in $40 \mathrm{~mL}$ dry toluene. A $850 \mu \mathrm{L}$ trimethoxy(3,3,3-trifluoropropyl) silane, $\mathrm{CF}_{3} \mathrm{CH}_{2} \mathrm{CH}_{2} \mathrm{Si}(\mathrm{OMe})_{3}$, was added dropwise into the above solution. The mixture was then treated at $80{ }^{\circ} \mathrm{C}$ in the argon atmosphere under stirring for $24 \mathrm{hrs}$. The solids were separated by centrifugation, washed with ethanol for three times, and dried under vacuum. The morphology of the pore structure retains, as shown in Figure $\mathrm{S} 3 \mathrm{a}, \mathrm{b}$. The pore sizes of the $\mathrm{CF}_{3}$ - functionalized core nanocatalysts are only slightly decrease compared to the bare one, which confirms that functional groups do not block mesoporous pores (Figure S4b, Table S1).

\section{S1.3. Synthesis of $\mathrm{SO}_{3} \mathrm{H}$-functionalized core-shell nanocatalysts by post-synthesis method}

The $\mathrm{SO}_{3} \mathrm{H}$-functionalization was achieved by the oxidation of - $\mathrm{SH}$ functional groups. In a typical synthesis, $80 \mathrm{mg}$ bare core-shell nanocatalysts with a $3 \mathrm{~nm}$ pore size was dispersed in $27 \mathrm{~mL}$ dry toluene and refluxed under argon atmosphere for $1 \mathrm{hr} .730 \mu \mathrm{L}$ (3-mercaptopropyl)trimethoxy silane, $\mathrm{HSCH}_{2} \mathrm{CH}_{2} \mathrm{CH}_{2} \mathrm{Si}(\mathrm{OMe})_{3}$, in $2 \mathrm{~mL}$ dry toluene was added dropwise into the above solution, and the mixture was maintained at $120{ }^{\circ} \mathrm{C}$ under reflux for $48 \mathrm{hrs}$. The solids were separated by centrifugation, washed with ethanol three times, and dried under vacuum. SH-functionalized coreshell nanocatalysts were prepared. In the next step, around $40 \mathrm{mg}$ of SH-functionalized core-shell nanocatalysts were dispersed in $100 \mathrm{~mL}$ aqueous $2 \mathrm{M} \mathrm{HCl}(16 \mathrm{~mL}$ concentrated $\mathrm{HCl}$ with $84 \mathrm{~mL}$ 
water). $50 \mathrm{~g} 30$ wt. $\% \mathrm{H}_{2} \mathrm{O}_{2}$ was then added slowly into the above solution, and the mixture was stirred at room temperature for $24 \mathrm{hrs}$ to fully oxidized/protonate the $-\mathrm{SH}$ group to $-\mathrm{SO}_{3} \mathrm{H}$ group. To ensure complete conversion of - $\mathrm{SH}$ group to $-\mathrm{SO}_{3} \mathrm{H}$ group, we used a large excess $\mathrm{H}_{2} \mathrm{O}_{2}$. The solids were separated by centrifugation, washed with ethanol three times, and dried under vacuum. The morphology of the pore structure retains, as shown in Figure S3c, d. The pore sizes of the $\mathrm{SO}_{3} \mathrm{H}$ - functionalized core nanocatalysts only slightly decrease compared to the bare one confirming that functional groups do not block mesoporous pores (Figure S4c, Table S1). 

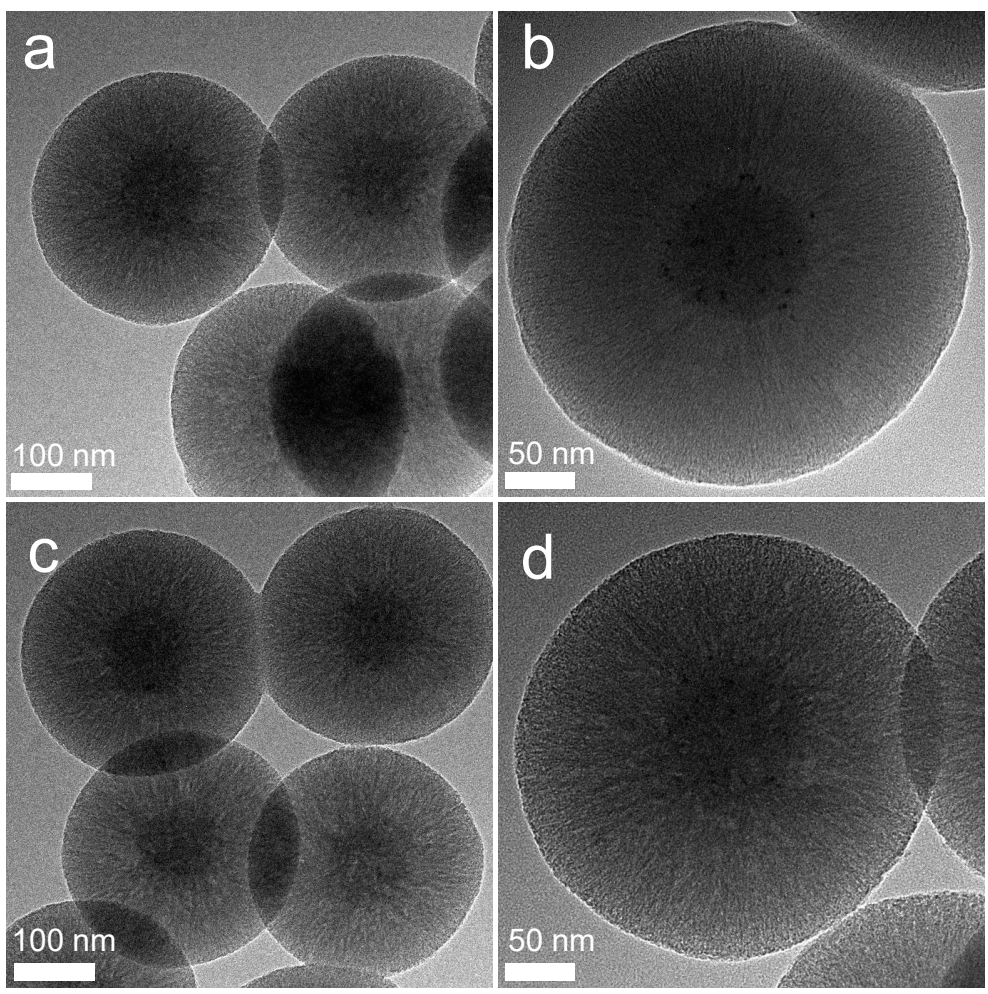

Figure S3. TEM images of nanocatalysts with $-\mathrm{CF}_{3}(\mathrm{a}, \mathrm{b})$ and $-\mathrm{SO}_{3} \mathrm{H}(\mathrm{c}, \mathrm{d})$ functionalization.
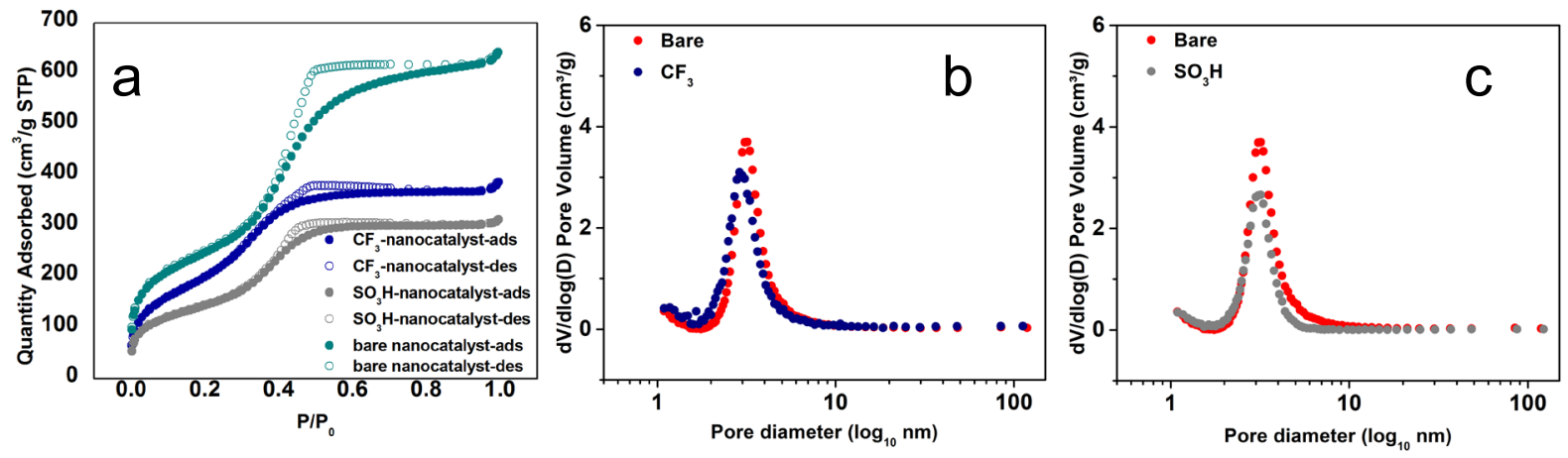

Figure S4. (a) $\mathrm{N}_{2}$ sorption isotherm of $\mathrm{CF}_{3}$ - and $\mathrm{SO}_{3} \mathrm{H}$-functionalized nanocatalyst and BJH pore size distributions of nanocatalysts with $-\mathrm{CF}_{3}(\mathrm{~b})$ and $-\mathrm{SO}_{3} \mathrm{H}$ (c) functionalization.

Table S1. Porosity information of $\mathrm{CF}_{3}-$ and $\mathrm{SO}_{3} \mathrm{H}-$ functionalized nanocatalyst.

\begin{tabular}{|c|c|c|}
\hline sample & $\begin{array}{l}\text { BET surface area } \\
\left(\mathrm{m}^{2} / \mathrm{g}\right)\end{array}$ & Pore size (nm) \\
\hline $\mathrm{SO}_{3} \mathrm{H}$-nanocatalyst & 515 & 3.1 \\
\hline CF3-nanocatalyst & 716 & 2.8 \\
\hline Bare-nanocatalyst & 860 & 3.4 \\
\hline
\end{tabular}


S1.4. Characterization and quantification of $\mathrm{CF}_{3}$ - and $\mathrm{SO}_{3} \mathrm{H}$ - functional group in core-shell nanocatalysts

The presence of the functionalized groups in core-shell nanocatalysts synthesized by the postfunctionalization method was confirmed by the DRIFTS studies with respective C-F and S-O vibrations being identified in Figure $\mathrm{S} 5 \mathrm{a}$, $\mathrm{b}$. The presence of the $-\mathrm{CF}_{3}$ group and the $-\mathrm{SO}_{3} \mathrm{H}$ group is evidenced by the $-\mathrm{CF}_{3}\left(1210 \mathrm{~cm}^{-1}\right)^{2}$, and asymmetric stretching of $-\mathrm{SO}_{2^{-}}\left(\sim 1340 \mathrm{~cm}^{-1}\right)^{3-4}$ vibrations as reported in the literature. Moreover, the surface functional groups were quantified by ${ }^{29} \mathrm{Si}$ solid-state NMR (SSNMR), as summarized in Figure S5c, d. ${ }^{5}$ The directly polarized ${ }^{29} \mathrm{Si}$ SSNMR spectra were obtained on a Chemagnetics $400 \mathrm{MHz}$ spectrometer, equipped with a 5-mm
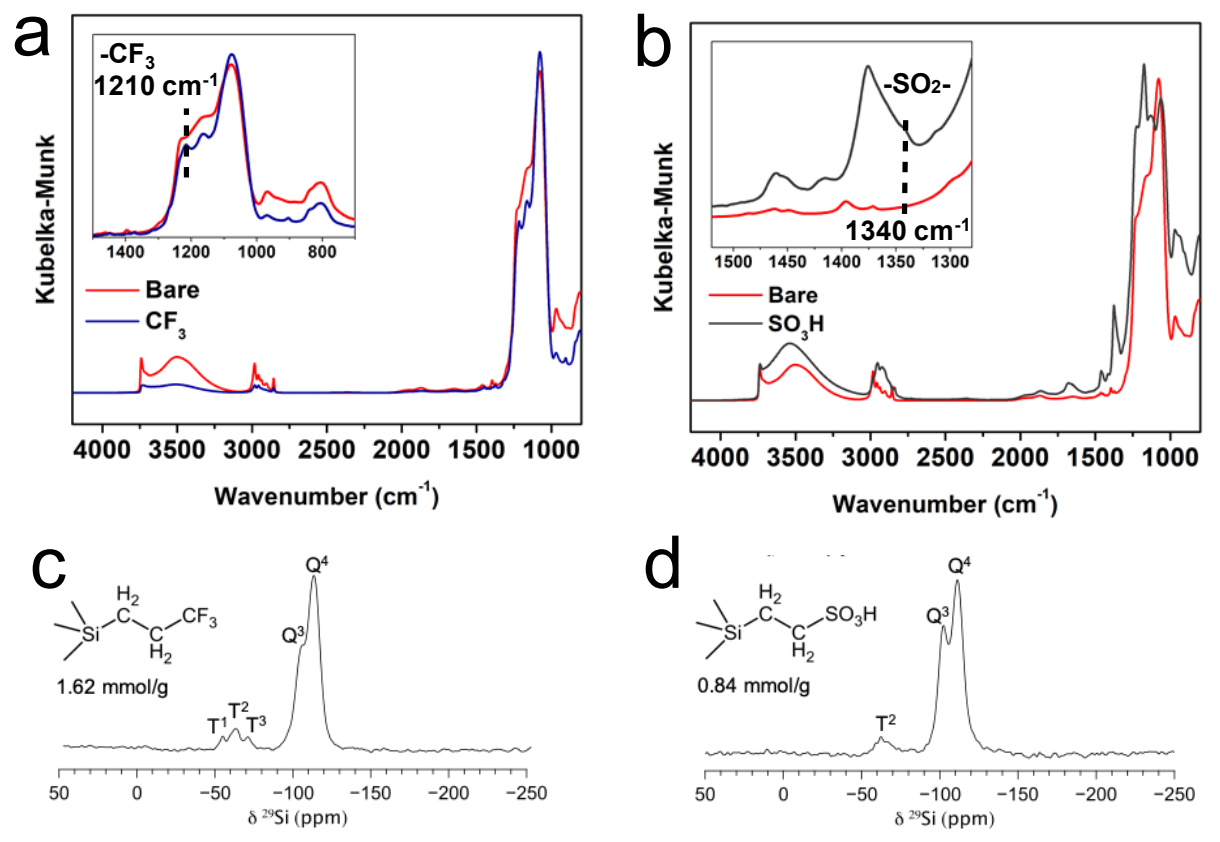

Figure S5. Characterization of the functionalization on silica surface. DRIFTS spectra of (a) $\mathrm{CF}_{3}$-core-shell and (b) $\mathrm{SO}_{3} \mathrm{H}$-core-shell nanocatalysts prepared by post-synthesis method. ${ }^{29} \mathrm{Si}$ SSNMR spectra of (c) $\mathrm{CF}_{3}$-core-shell and (d) $\mathrm{SO}_{3} \mathrm{H}$-core-shell nanocatalysts prepared by post-synthesis method. The resonances are assigned as follows: $\mathrm{Q}^{4}-(\equiv \mathrm{SiO})_{4} \mathrm{Si} ; \mathrm{Q}^{3}-(\equiv$ $\mathrm{SiO})_{3} \mathrm{SiOH} ; \mathrm{T}^{3}$ - $(\equiv \mathrm{SiO})_{3} \mathrm{SiR} ; \mathrm{T}^{2}$ - $(\equiv \mathrm{SiO})_{2} \mathrm{Si}(\mathrm{OH}) \mathrm{R}$ and $\mathrm{T}^{1}-(\equiv \mathrm{SiO}) \mathrm{Si}(\mathrm{OH})_{2} \mathrm{R}$. 
magic angle spinning probe. The spectral intensities, resulting from acquisition of 512 scans with a recycle delay of $300 \mathrm{~s}$, yielded the quantities of surface functional groups of $1.62( \pm 0.15)$ and $0.84( \pm 0.15) \mathrm{mmol} / \mathrm{g}$ for $\mathrm{CF}_{3}$ - and $\mathrm{SO}_{3} \mathrm{H}$-functionalized nanocatalysts, respectively. The surface densities of functional groups are respective 1.3, 0.97 per $\mathrm{nm}^{2}$ for $\mathrm{CF}_{3}$ - and $\mathrm{SO}_{3} \mathrm{H}$-functionalized core-shell nanocatalysts as their surface areas measured as 740 and $520 \mathrm{~m}^{2} / \mathrm{g}$. We noticed that the theoretical silanol density is $3.9-9.1 \mathrm{mmol} / \mathrm{g}\left(3-7 \mathrm{per} \mathrm{nm}^{2}\right)$ estimated based on the bare mesoporous silica with the surface area of $800 \mathrm{~m}^{2} / \mathrm{g}$. The surface functional groups is not capable of passivating all silanol groups in one batch of the post-functionalization method, even though we added the stoichiometric amount of silane precursors adequate to cap all free silanol groups. Using X-ray

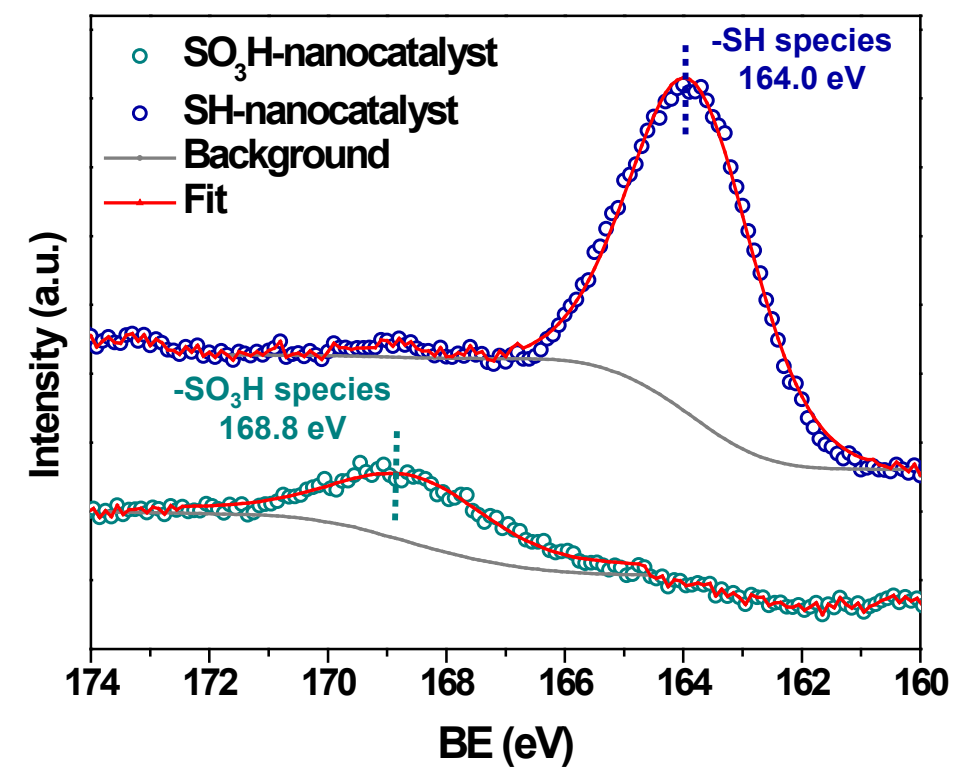

Figure S6. XPS spectra of S2p in SH- and $\mathrm{SO}_{3} \mathrm{H}$-functionalized nanocatalysts.

photoelectron spectroscopy (XPS), we further confirmed the oxidation of $-\mathrm{SH}$ to $-\mathrm{SO}_{3} \mathrm{H}$ by $\mathrm{H}_{2} \mathrm{O}_{2}$, where the binding energy of S2p shifts from $164.0 \mathrm{eV}$ to $168.8 \mathrm{eV}$ (Figure S6) ${ }^{6-8} \mathrm{XPS}$ spectrum was determined by the PHI 5500 Multi-technique system (Physical Electronics, Chanhassen, MN) equipped with a mono-chromatized $\mathrm{Al} K_{\alpha} \mathrm{X}$-ray source $(1486.6 \mathrm{eV})$. 


\section{S1.5. Reagents used in the synthesis of nanocatalysts}

$\mathrm{K}_{2} \mathrm{PtCl}_{4}$ (Acros Organics), $\mathrm{NH}_{3} \cdot \mathrm{H}_{2} \mathrm{O}$ (EMD), ethanol (200 proof, local vendor), cyclohexane (Fisher Scientific), L-arginine (Sigma-Aldrich), tetraethyl orthosilicate (Sigma-Aldrich), tetradecyltrimethylammonium bromide (Sigma-Aldrich), polyvinylpyrrolidone (PVP-K30, $\mathrm{Mw}=40,000$, Sigma-Aldrich), ethylene glycol (EG, Fisher Scientific), hexane (Fisher Scientific), isopropanol (Fisher Scientific), (3-aminopropyl) triethoxysilane (Alfa Aesar), hexadecyltrimethylammonium bromide (Sigma-Aldrich), methanol (Fisher Scientific), (3mercaptopropyl)trimethoxy silane (Sigma), toluene (Fisher Scientific), concentrated $\mathrm{HCl}$ (trace metal grade, Fisher Scientific), $\mathrm{H}_{2} \mathrm{O}_{2}$ (30 wt.\%, Fisher Scientific), trimethoxy(3,3,3-trifluoropropyl) silane (Alfa Aesar). 


\section{S2. Single-particle single-molecule fluorescence experiments.}

\section{S2.1. Sample preparation.}

The core-shell nanocatalysts were dispersed in ultra-pure methanol and diluted several times to appropriate concentrations in $1.5 \mathrm{~mL}$ micro-centrifuge tubes. The nanocatalyst solutions were then sonicated in a water bath for $15 \mathrm{~min}$ at room temperature. The sonication process is necessary for achieving well dispersed single nanocatalysts in solution. After sonication, the nanocatalysts solution was centrifuged at $1 \times \mathrm{g}$ speed for 1 minute. Aggregated nanocatalysts are more massive



Figure S7. Bright field image of core-shell nanocatalysts on quartz slide surface. The bright field image was captured under the same setup as imaging fluorescence but without inserting the emission filters into the light path of the microscope. The well separated spots in the image indicate the catalytic reaction study on single nanocatalysts.

than single nanocatalysts; thus, they will be moved toward the bottom of microcentrifuge tubes during the centrifuging process. We then use about $10 \mu \mathrm{L}$ of the supernatant solution to drop-cast nanocatalysts on quartz slides. Using this procedure, we can achieve well-dispersed single nanocatalysts on a quartz slide surface (Figure S7).

In order to minimize fluorescence impurities and background, quartz slides were cleaned in piranha solution, sonicated in Mili-Q water, and plasma cleaned before drop-casting nanocatalysts 
on them. We assembled quartz slides and coverslips to a flow chamber using double-sided tape. Quartz slides were pre-drilled with two holes for inserting tubing. Epoxy glue was used to secure the tubing and seal the gap. During single particle single-molecule imaging experiments, a $1 \times$ PBS buffer solution $(\mathrm{pH}=7.4$, Invitrogen) containing reactant molecules Amplex red (Invitrogen) and oxidative reagent hydrogen peroxide (Sigma-Aldrich) was introduced to the nanocatalysts through the flow chamber using a syringe pump (Harvard Apparatus) with a flow rate of $20 \mu \mathrm{L} \mathrm{min}{ }^{-1}$. The concentration of Amplex red was from 0.02 to $10 \mu \mathrm{M}$, while the hydrogen peroxide was kept at a saturated concentration of $10 \mathrm{mM}$.

\section{S2.2. The imaging set-up.}

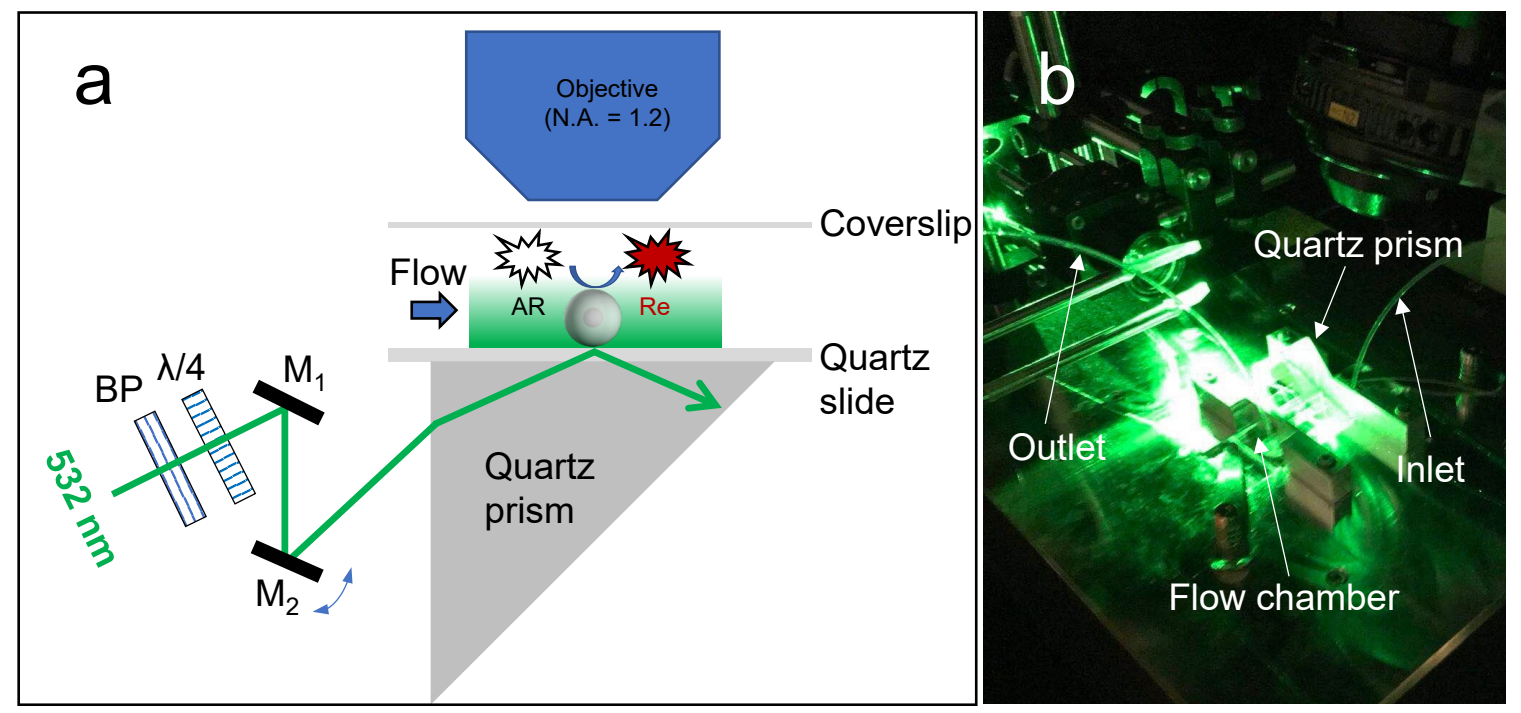

Figure S8. Prism-type total internal reflection fluorescence microscopy for single particle single-molecule fluorescence experiments. (a) schematic view and (b) a picture of experimental setup. BP: laser clean up filter (LL01-532-12.5, Semrock), $\lambda / 4$ : quarter waveplate (WPQ05M-532, Thorlabs), M: reflecting mirror.

Single particle single-molecule imaging experiments were carried out on a prism-type total internal reflection fluorescence (TIRF) system integrated into a Nikon Ti-E inverted microscope (Figure S8). The generated resorufin product molecule was excited by focusing a circularly polarized 532 $\mathrm{nm}$ laser beam $(15 \mathrm{~mW})$ on the sample with a spot size of $100 \mu \mathrm{m} \times 80 \mu \mathrm{m}$. The fluorescence 
emission signals from resorufin molecules are collected by $60 \times$ high numerical aperture (N.A. $=$ 1.2) water immersion objective (Olympus) and imaged on Andor iXonEM ${ }^{+}$Ultra 888 EMCCD camera (Belfast, Northern Ireland) at a temporal resolution of $30 \mathrm{~ms}$. A filter set composed of bandpass filter 607/70 (Semrock) and notch filter 532 (Semrock) was inserted before the camera to block the fluorescence impurities and scattering signal from the excitation laser. The collected movies and images were analyzed using a self-written MATLAB script. 
S2.3. Quantify reaction dynamics at single particle single-molecule level with turn-over resolution.
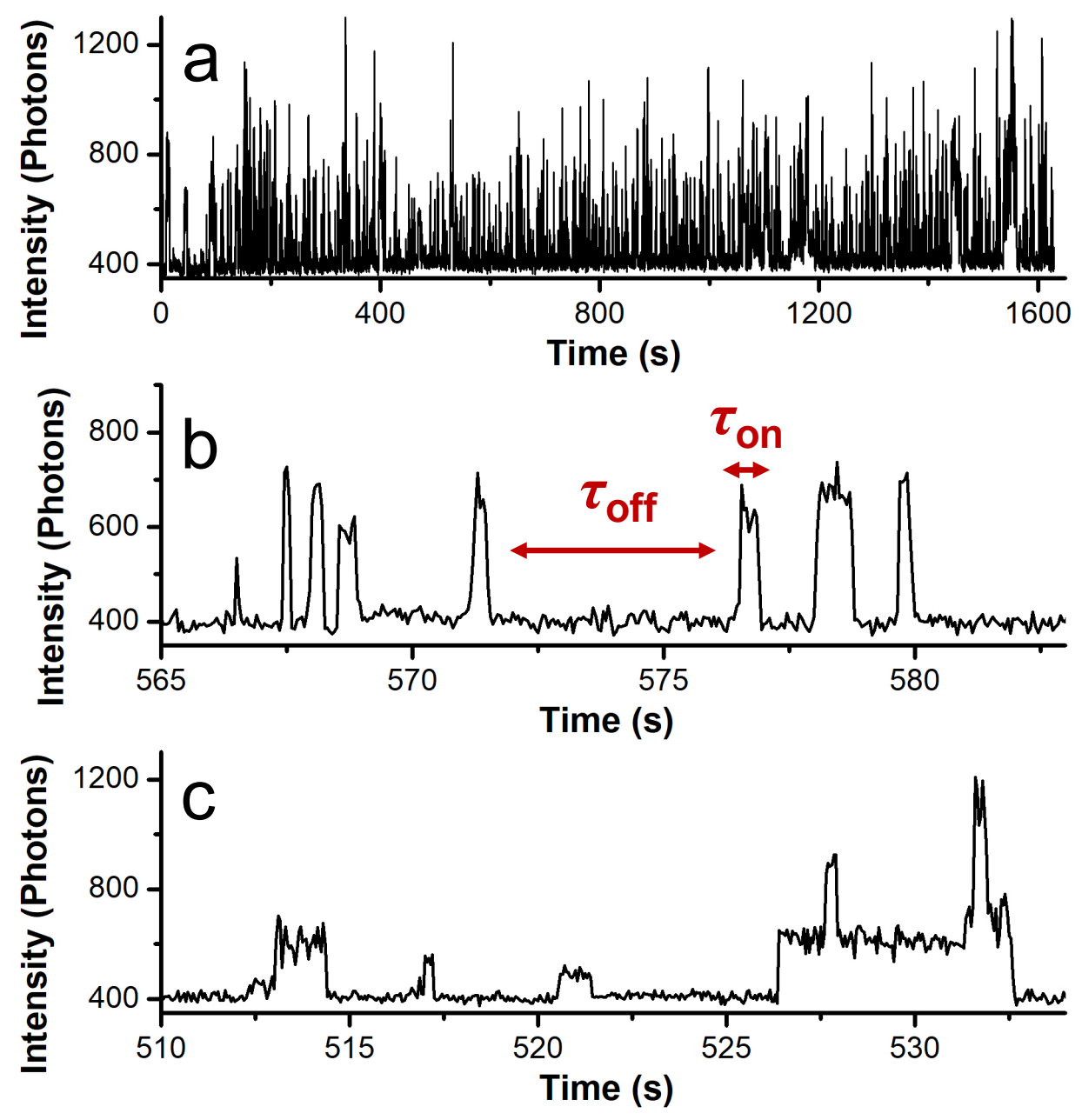

Figure S9. Single-molecule imaging of catalytic reactions on single nanocatalysts. (a) a typical of fluorescence intensity trajectory on a single nanocatalysts over an imaging period of $\sim 27$ min. (b) Segment of fluorescence intensity trajectory on a single nanocatalysts. $\tau_{\text {off }}$ and $\tau_{\text {on }}$ were determined at turn-over resolution. (c) Segment of fluorescence intensity trajectory showing that multiple catalytic reactions could happen within the same on-off cycle.

Figure S9a shows a typical fluorescence intensity trajectory on a single nanoparticle. The on-off of fluorescence intensity is associated with the catalytic reaction. Every one of the fluorescent bursts represents a catalytic event. We can measure the catalytic reaction activities by counting these individual reaction events. Two waiting times can be attributed from the fluorescence intensity trajectory: $\tau_{\text {off }}$ is the waiting time between consecutive catalytic events reflecting the time 
for product formation, and $\tau_{\mathrm{on}}$ is the waiting time for product molecule dissociation after formation (Figure S9b). $\tau_{\text {off }}$ can be used to determine the catalytic reaction rates. $\tau_{\text {on }}$ can be used to determine the dissociation rates of product molecules. Longer $\tau_{\text {off }}$ and $\tau_{\text {on }}$ means slower catalytic reaction rate and slower dissociation rate, respectively. During the imaging experiments, we also found out that multiple catalytic reactions could happen within the same on-off cycle (Figure S9c). It is impossible to differentiate individual catalytic events in these cases. Therefore, we excluded these types of catalytic events from quantitative analysis of reaction kinetics. Fitting the distribution of $\tau_{\text {off }}$ using exponential decay function (Figure S10a) gives the characteristic average value, $<\tau_{\text {off }}>$. 9 The catalytic reaction rate $v_{\mathrm{r}}$ is determined as $\left\langle\tau_{\text {off }}\right\rangle^{-1}$, which is $0.24 \pm 0.04 \mathrm{~s}^{-1}$ particle $^{-1}$ for this specific example. Fitting the distribution of $\tau_{\text {on }}$ using exponential decay function (Figure S10b) gives the characteristic average value, $<\tau_{\mathrm{on}}>$. The dissociation rate $v_{\mathrm{d}}$ is determined as $<\tau_{\mathrm{on}}>^{-1}$, which is $3.8 \pm 0.1 \mathrm{~s}^{-1}$ particle $^{-1}$ for this specific example.
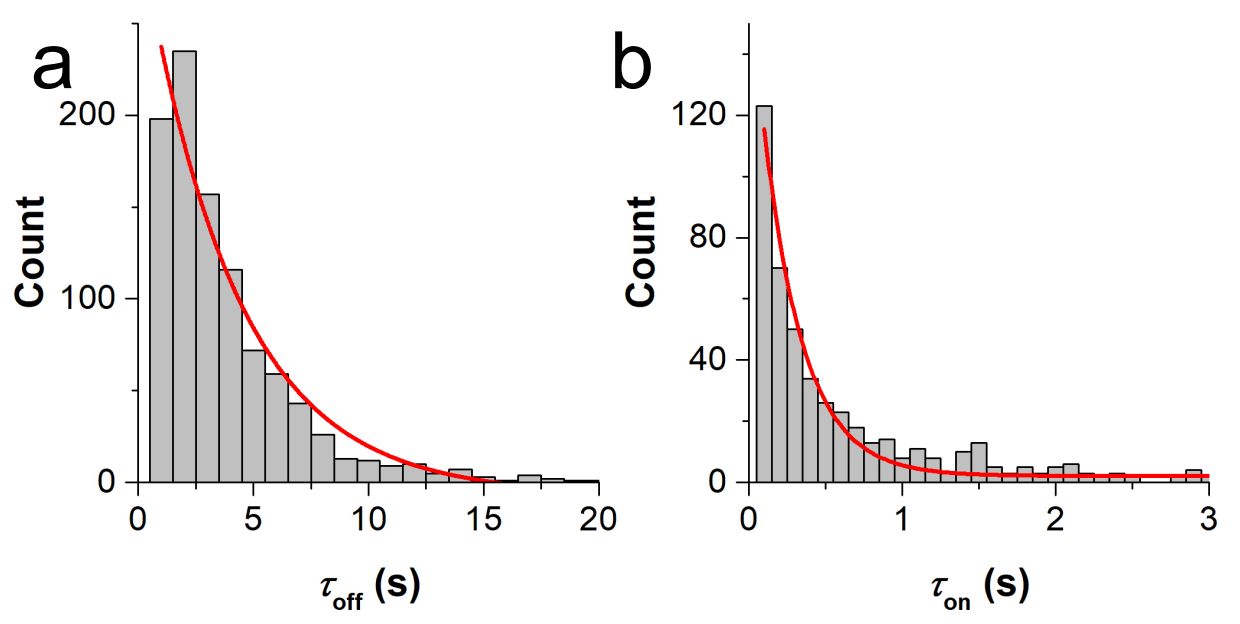

Figure S10. Quantitative analysis of catalytic reaction rate and product dissociation rate. (a) Fitting the distribution of $\tau_{\text {off }}$ using exponential decay function gives the characteristic average value, $\left\langle\tau_{\text {off }}>\right.$, of $4.1 \pm 0.6$ s. (b) Fitting the distribution of $\tau_{\text {on }}$ using exponential decay function gives the characteristic average value, $<\tau_{\text {on }}>$, of $0.26 \pm 0.01 \mathrm{~s}$. 


\section{S3. Ensemble measurement of catalytic activities.}

The effects of nanopore hydrophobicity on the catalytic activities were also tested using ensemble measurements. A fluorometer was used to measure the fluorescence intensity of the resorufin product molecule at different time points. The fluorescence emission signal from the product
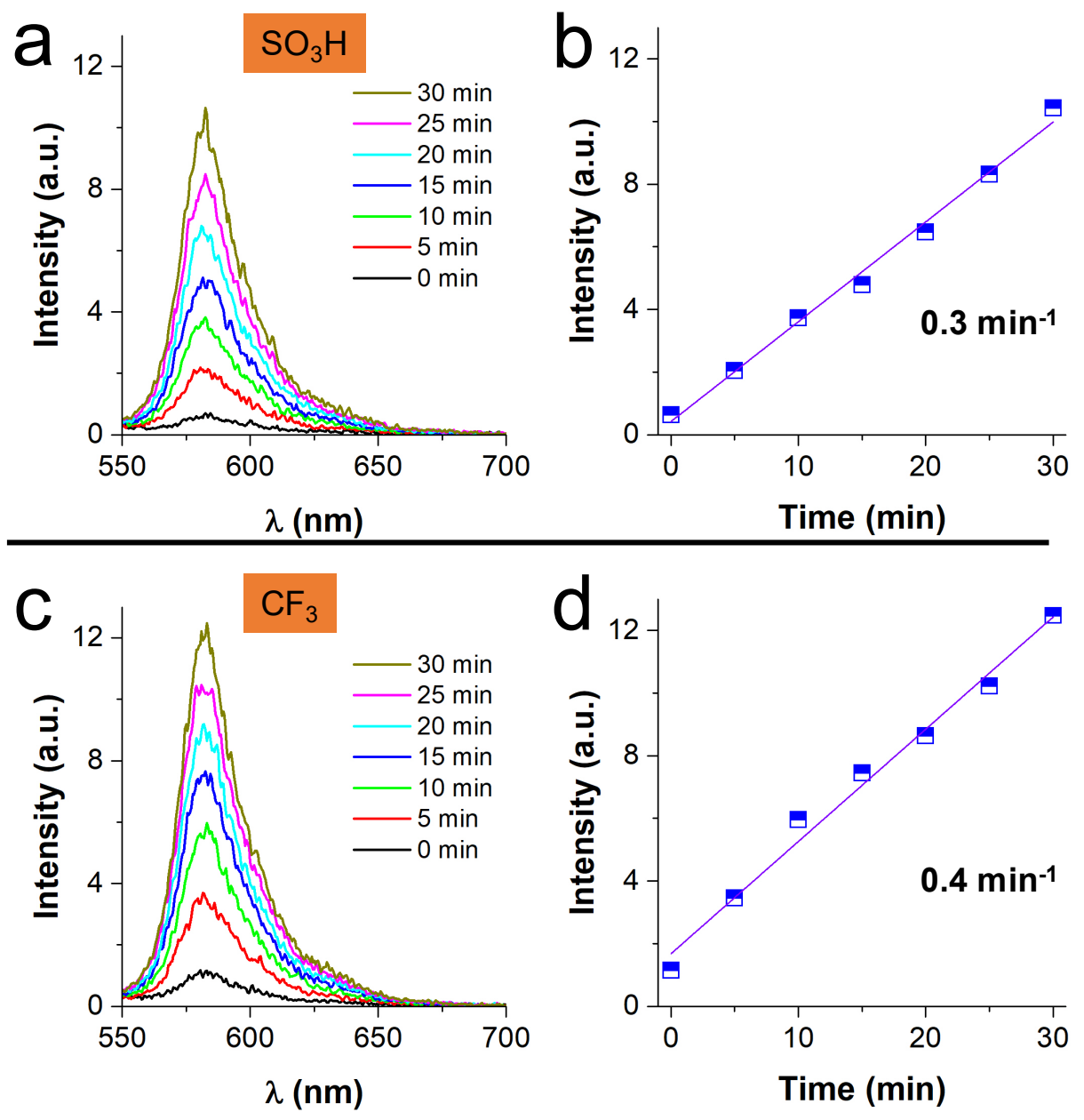

Figure S11. Ensemble measurement of catalytic activities. Time-lapse fluorescence emission spectra of resorufin product molecules generated during catalytic reactions at room temperature in hydrophilic (a) and hydrophobic (c) nanopores. Fluorescence intensity at 583 $\mathrm{nm}$ over time for catalytic reactions in hydrophilic (b) and hydrophobic (d) nanopores. Same concentration of two types of nanocatalysts was used in the experiments.

resorufin at $583 \mathrm{~nm}$ was measured every five minutes for a 30-minute duration for each of our catalysts under the same reaction conditions $\left(10 \mu \mathrm{M}\right.$ Amplex red, $10 \mathrm{mM} \mathrm{H}_{2} \mathrm{O}_{2}$ in $1 \times \mathrm{PBS}$ buffer, 
$\mathrm{pH}$ 7.4). As shown in Figure S11, the activities of the catalysts with a hydrophobic functional group $\left(\mathrm{CF}_{3}\right)$ were higher than the catalysts with a hydrophilic functional group $\left(\mathrm{SO}_{3} \mathrm{H}\right)$, which agrees with the results from single-molecule single-particle catalysis experiments. Both singlemolecule and ensemble data support the increase of catalytic activity of Pt NPs with a hydrophobic pore environment. 


\section{S4. Molecular orientation in nanopores.}

AR molecule has a rigid planar structure with a molecular size of $1.3 \times 0.7 \mathrm{~nm}$. The nanopore size

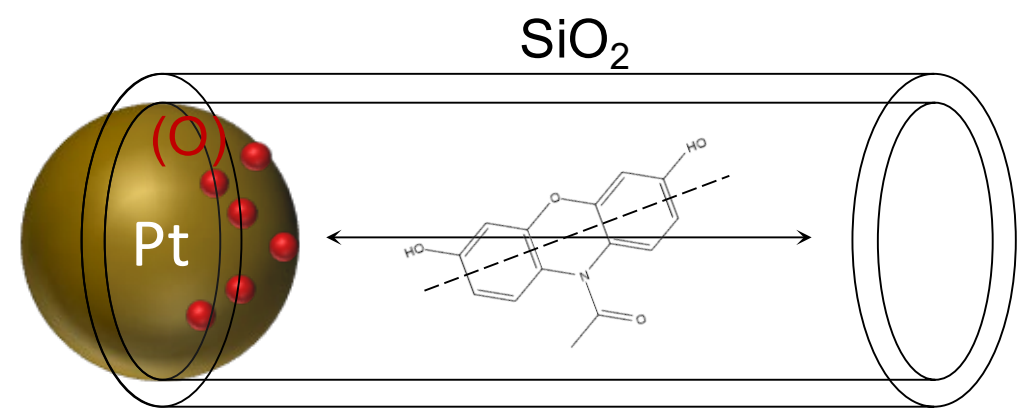

Figure S12. Molecular orientation in nanopores.

in our experiments is $\sim 3 \mathrm{~nm}$. The molecular length of AR is larger than $1 / 3$ of the physical size of nanopores. It has been shown that planar molecules like AR would be oriented with its long molecular axis parallel to the pore direction (Figure S12) when they are confined in such small space. ${ }^{10-14}$ The molecular orientations are determined by factors including molecular length, pore diameter, surface property of pore. Furthermore, AR molecules would approach to catalytic centers Pt NPs with one end of the long molecular axis facing toward to Pt NPs surface. Both theoretical studies and experimental measurements suggest that aromatic molecules tend to adsorb flat on the metal surfaces by forming pi-bonds with the metal surface. ${ }^{15-19}$ Weaker adsorption strength will be presented when the AR approach to Pt NPs surface with such tilted orientations. 


\section{S5. Reaction mechanisms of the oxidation of Amplex red.}

Platinum (Pt) nanoparticle catalyzed oxidation of non-fluorescent Amplex red to produce highly fluorescent resorufin by hydrogen peroxide follows a reaction mechanism of two-step singleelectron transfer process. ${ }^{1,20-21}$ In the initial step, the phenol group of Amplex red reacts with the

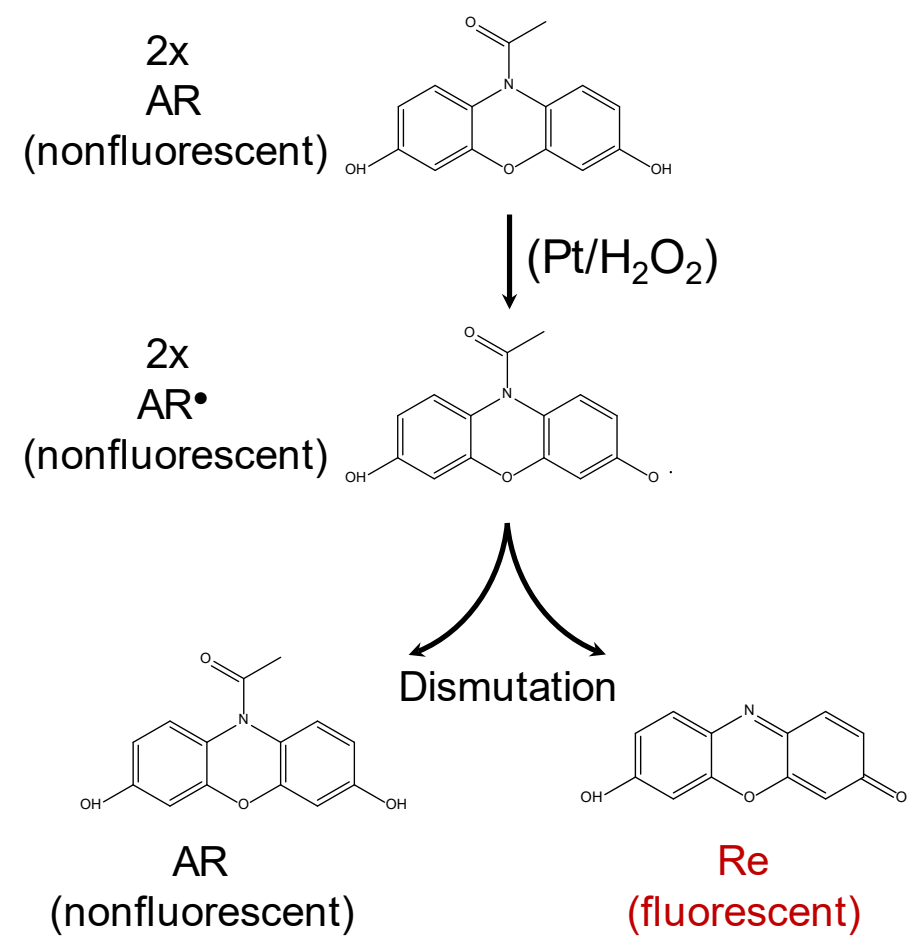

Figure S13. Reaction mechanism of the oxidation of Amplex red to resorufin.

chemisorbed oxygen on Pt nanoparticles ${ }^{22}$ to generate intermediate species: Amplex red radicals. The follow-up dismutation reaction requires two Amplex red radicals and will generate one Amplex red molecule and one resorufin molecule. The resorufin molecule is the final product of the catalytic reaction. 


\section{S6. Ensemble measurement of activation energy of catalytic reaction in nanopores.}

Temperature controlled ensemble experiments were also conducted to study the effect of the nanopore hydrophobicity on the activation energy of the catalytic reactions. The experiments were
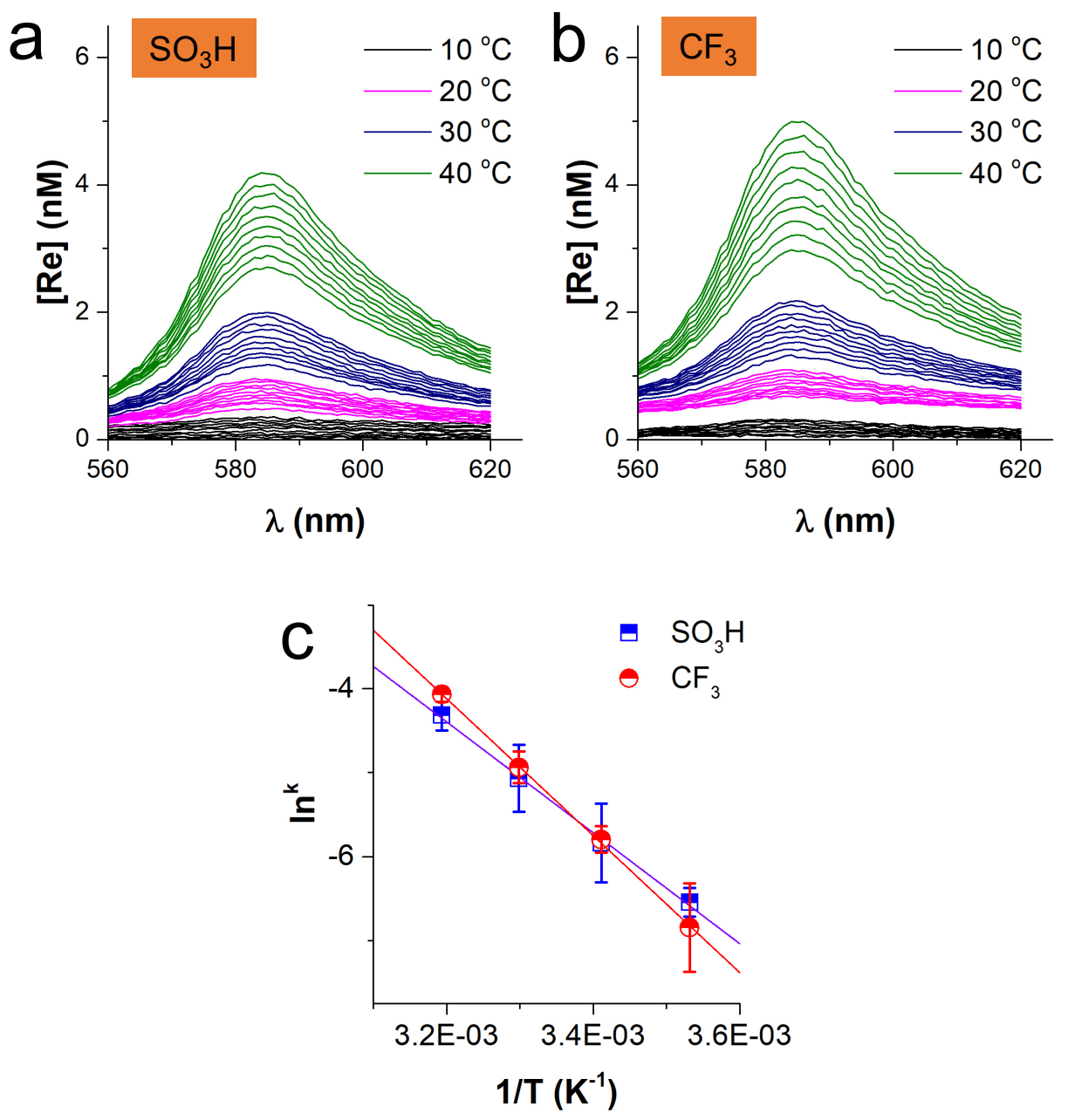

Figure S14. Ensemble measurement of activation energy of the oxidation of AR catalyzed by $\mathrm{SiO}_{2} @ \mathbf{P t} @ \mathbf{m S i O}_{2}$. Concentrations of product molecule resorufin (Re) during 20 minutes of catalytic reactions in hydrophilic nanopores (a) and hydrophobic nanopores (b) at controlled temperatures from $10{ }^{\circ} \mathrm{C}$ to $40{ }^{\circ} \mathrm{C}$. (c) Natural $\log$ of reaction rate constants $\left(\ln ^{k}\right)$ over the inverse of absolute temperatures $(1 / T)$.

conducted using Jasco J-1500 spectrophotometer. The sample was composed of $1 \times$ PBS buffer, our core-shell nanocatalyst, reactant Amplex red $(20 \mu \mathrm{M})$, and oxidative reagent hydrogen peroxide $(100 \mathrm{mM})$. Saturated reactant concentration $(20 \mu \mathrm{M})$ was used in the measurement, 
giving reaction rate constant equals to the determined reaction rate. High concentrations of Amplex red and hydrogen peroxide were used to ensure the reactions were always taken under saturated conditions at different temperatures since the experiments took $2 \sim 3$ hours to finish. The sample was placed in a cuvette and stirred at $800 \mathrm{rpm}$ during measurements. The fluorescence emission spectra (Figure S14a, b) from product molecule resorufin was recorded every 2 minutes for 20 minutes at each of four temperatures $\left(10,20,30\right.$, and $\left.40^{\circ} \mathrm{C}\right)$.

By varying the reaction temperature, we can measure the activation energy $\left(E_{a}\right)$ by analyzing both the temperature- and time-dependent fluorescence spectrum of the substrate. Reaction rates at four temperatures were obtained from the slope of the time versus the fluorescence intensity of the product resorufin molecules. To calculate the activation energies, we used the Arrhenius equation $k=A \exp \left(-E_{a} / R T\right)$ where $k$ is the rate constant, $A$ is the effective collision factor, $R$ (8.314 $\mathrm{J} \mathrm{K}^{-1} \mathrm{~mol}^{-1}$ ) is the ideal gas constant and $T$ is the absolute temperature in kelvin. The Arrhenius plot $\left(\ln { }^{k}\right.$ versus $\left.1 / T\right)$ was linearly fitted to yield the activation energy $E_{a}$ for each nanocatalyst. As seen from the results in Figure S14c, the catalytic reaction in hydrophobic nanopores $(67 \pm 1 \mathrm{~kJ}$ $\left.\mathrm{mol}^{-1}\right)$ has higher activation energy than that in hydrophilic nanopores $\left(56 \pm 2 \mathrm{~kJ} \mathrm{~mol}^{-1}\right)$. 


\section{S7. Single-molecule tracking in nanopores}

S7.1. Super-localization of the center positions of single-molecule images.
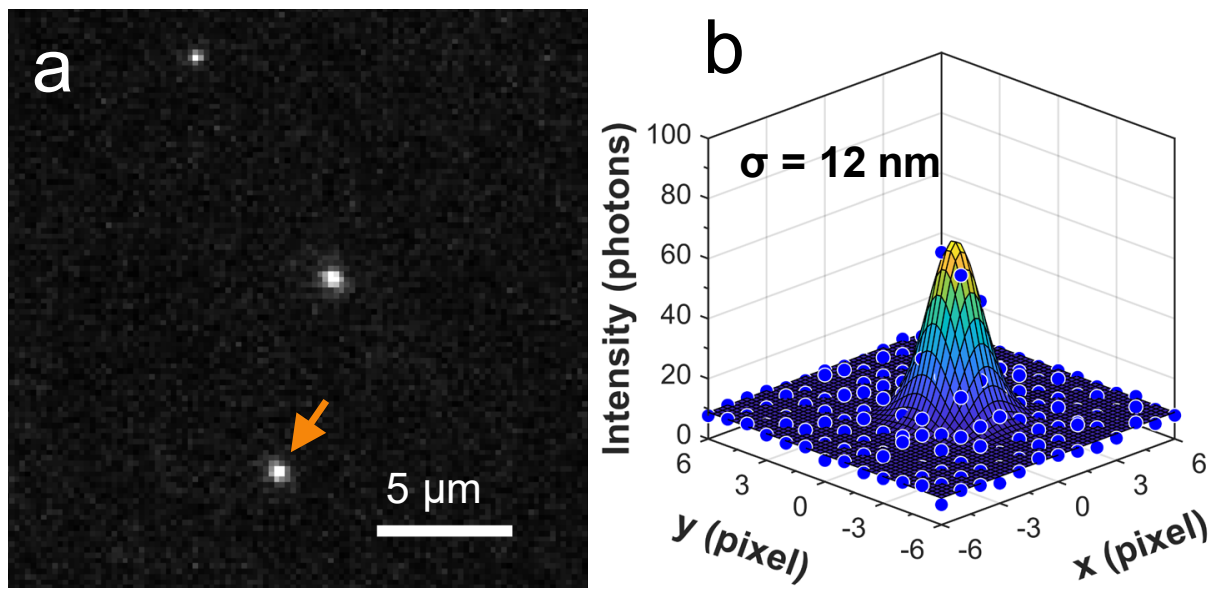

Figure S15. Super-localize the center position of single molecule images. (a) Images of single molecules generated during the catalytic reaction on single nanocatalysts. (b) Gaussian fitting the intensity distribution of single-molecule image gives the molecular center position with $12 \mathrm{~nm}$ localization precision.

Figure $\mathrm{S} 15 \mathrm{~b}$ is the fluorescence intensity distribution in the 2D space of the molecule highlighted in Figure S15a. The intensity distribution was fitted using an elliptical Gaussian function $I_{x y}=$ $A+B * e^{\left(\frac{\left(x-x_{0}\right)^{2}}{2 S_{x}^{2}}+\frac{\left(y-y_{0}\right)^{2}}{2 S_{y}^{2}}\right)}$ where $A$ is the background level intensity, $B$ is the maximum intensity, $\left(x_{0}, y_{0}\right)$ is the center locations of the molecule, and $S_{x}, S_{y}$ are the standard deviation of the Gaussian distribution in $\mathrm{x}, \mathrm{y}$ that equal $1 / 2.2$ of the PSF width. The localization precision is determined using the following equation $\sigma_{x y}=\sqrt{\frac{S_{x y}{ }^{2}}{N}+\frac{a^{2}}{12 N}+\frac{8 \pi b^{2} S_{x y}{ }^{4}}{a^{2} N^{2}}}$ where $N$ is the number of collected photons, $a$ is the pixel size, and $b$ is background noise. The first term $\left(\frac{s_{x y}^{2}}{N}\right)$ is the photon noise (each photon collected in the image gives a measure of the position of the object, and the position error of each measurement is the same as the standard deviation of the point- spread function of the microscope). The best estimate of the position is then given by the average of the positions of the individual detected photons, with an error given by common statistical formula for the standard error on mean. 
The second term $\left(\frac{a^{2}}{12 N}\right)$ is the effect of the finite pixel size of the detector (arises from the uncertainty of where the photon arrived in the pixel. The extra noise adds to the uncertainty of each photon). The last term $\left(\frac{8 \pi b^{2} S_{x y}{ }^{4}}{a^{2} N^{2}}\right)$ is the effect of the background, which results from detected photons that do not originate from the particle. ${ }^{23}$ For the molecule highlighted in Figure S15a, the localization precision was determined to be $12 \mathrm{~nm}$ giving the parameters of calculated $S_{x y}=196$ $\mathrm{nm}, N=365$ photons, $b=2$ photons, and fixed pixel size $a=195 \mathrm{~nm}$.

\section{S7.2. Mean squared displacement analysis of single-molecule transport in nanopores.}

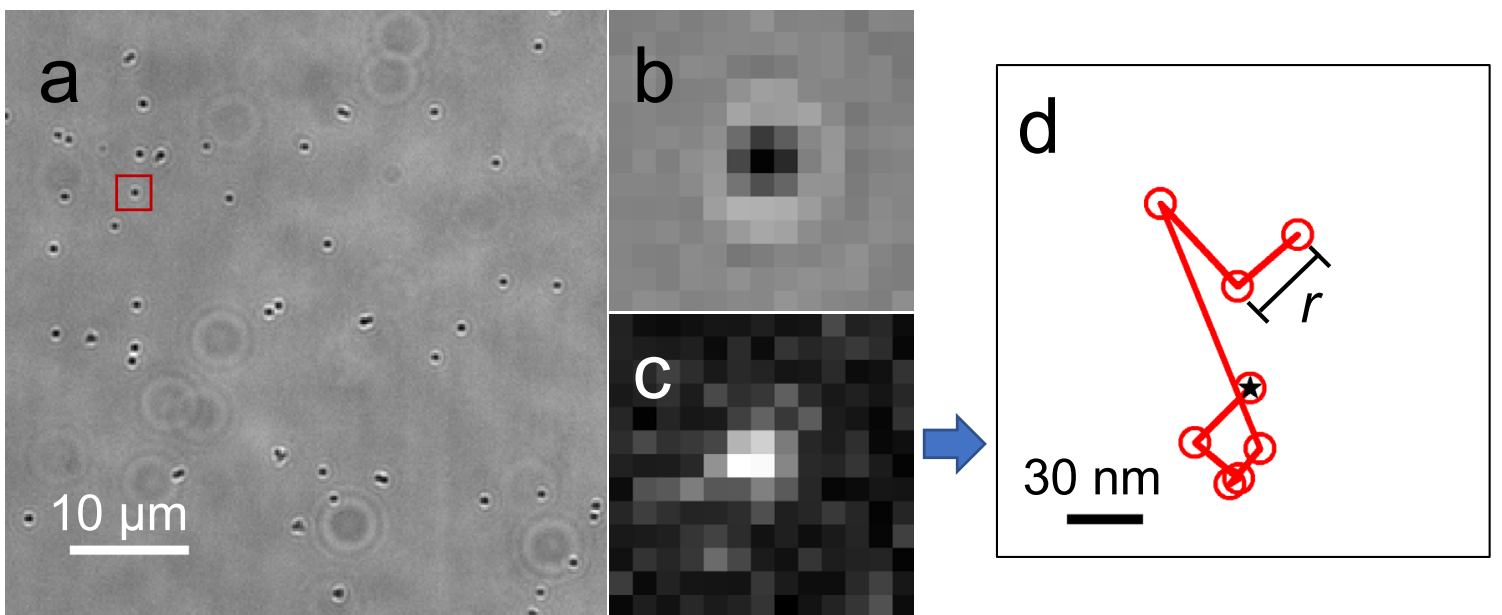

Figure S16. Typical single-molecule trajectories in nanopores. (a) Bright field (BF) microscopy images core-shell nanocatalyst on quartz slides. Zoom-in BF images (b) and fluorescence images (c) of generated single-molecule in catalytic reaction. (d) Typical singlemolecule trajectory during single catalytic turn-over events.

Representative trajectories of resorufin molecules inside the nanopores are shown in Figure S16 and analyzed using mean squared displacement (MSD) analysis. Because of the relatively short lifetime of resorufin molecules inside the nanopore, the global analysis strategy was used for achieving higher accuracy. Large datasets of single molecular trajectories are used to conclude with statistical significance. Figure S17a and c show the histogram distributions of the square root 
of displacement $(r)$ in resorufin molecular trajectories in hydrophilic and hydrophobic nanopores, respectively. The much larger average value of $r$ compared to the localization precision $\sigma$ (Figure S17b, d) suggests that resorufin is undergoing diffusive transport rather than permanently adsorbed at specific sites inside nanopores.
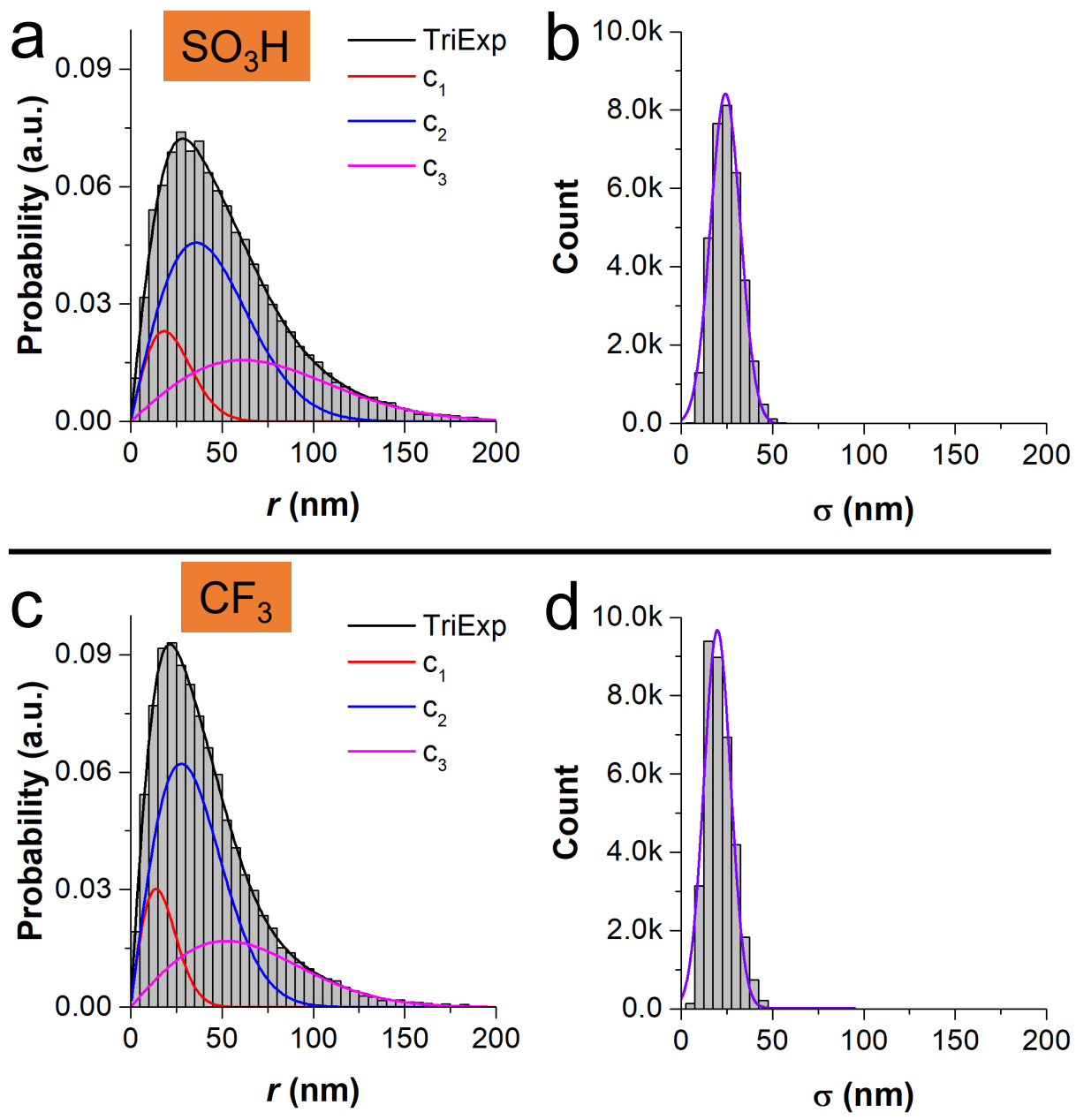

Figure S17. Mean squared displacement analysis of single molecular trajectories in nanopores. Histogram distribution of jump distance ( $r$ ) in hydrophilic (a) and hydrophobic (c) nanopores. Histogram distribution of localization precision $(\sigma)$ in hydrophilic (a) and hydrophobic (c) nanopores.

To quantitatively measure the molecular transport of resorufin inside the nanopore, we fitted the distribution of molecular displacement with the probability density function ${ }^{24}: p\left(r^{2}, t\right) \cdot d r^{2}=$ 
$\frac{1}{\pi\left\langle r_{(t)}^{2}\right\rangle} \exp \left(\frac{-r_{(t)}^{2}}{\left\langle r_{(t)}^{2}\right\rangle+\sigma^{2}}\right) 2 \pi r \cdot d r^{2}$ where $\left\langle r^{2}\right\rangle$ stands for the mean square displacement and $\sigma$ is the localization uncertainty. In heterogeneous environments, the molecular transport can be described by a multicomponent probability density function with distinct diffusion coefficients. $p\left(r^{2}, t\right)$. $d r^{2}=\sum_{1}^{i} c_{i} \frac{1}{\pi\left\langle r_{i(t)}^{2}\right\rangle} \exp \left(\frac{-r_{(t)}^{2}}{\left\langle r_{i(t)}^{2}\right\rangle+\sigma^{2}}\right) 2 \pi r \cdot d r^{2}$. The diffusion coefficient was determined based on the Einstein-Smoluchowski equation for random diffusion in one dimension $\left\langle r_{i(t)}^{2}\right\rangle=2 D_{i} \cdot t .^{25-26}$

To best describe the histogram distributions of $r$, it requires using radial probability density function with three components (denoted as $\mathrm{c}_{1}, \mathrm{c}_{2}, \mathrm{c}_{3}$, Figure $\mathrm{S} 17 \mathrm{a}, \mathrm{c}$ ) for fitting the data points resulting in three distinct characteristic diffusion coefficients $\left(D_{1}, D_{2}, D_{3}\right)$. The distinct diffusion coefficients indicate that the local environments inside nanopore are heterogeneous. Therefore, the transport of resorufin molecules in nanopores should be a combination of different motion modes, including surface adsorption and inner pore diffusion, instead of just a simple Brownian motion. The medium diffusion rate $\left(\mathrm{c}_{2}\right)$, which contains significant contributions from both $\mathrm{c}_{1}$ (adsorption) and $c_{3}$ (random movement), dominates the molecular transport. The apparent diffusion coefficient $D_{\text {app }}$ calculated as $\sum c_{i} D_{i}$ is smaller in hydrophobic nanopores $\left(0.016 \pm 0.003 \mu \mathrm{m}^{2} \mathrm{~s}^{-1}\right)$ than that in hydrophilic nanopores $\left(0.024 \pm 0.004 \mu \mathrm{m}^{2} \mathrm{~s}^{-1}\right)$. 


\section{S8. Enrichment of resorufin molecules in nanopores.}

The nanocatalysts are drop-cast on a quartz slide and assembled a flow cell. We then incubate these nanocatalysts with $\sim 1 \mu \mathrm{M}$ resorufin molecules for 1 hour. Fluorescence images of resorufin molecules inside the porous nanocatalysts were collected immediately after washing chamber once with $1 \times$ PBS buffer $(\mathrm{pH} 7.4)$. Typical fluorescence images of single nanocatalysts of hydrophilic $\left(\mathrm{SO}_{3} \mathrm{H}\right)$ and hydrophobic $\left(\mathrm{CF}_{3}\right)$ nanopores are shown in Figure $\mathrm{S} 18 \mathrm{a}, \mathrm{b}$, respectively. We measured fluorescence intensity over more than 100 nanocatalysts for both types and analyzed its distribution through histogram analysis (Figure S18c). Higher fluorescence intensity was obtained for
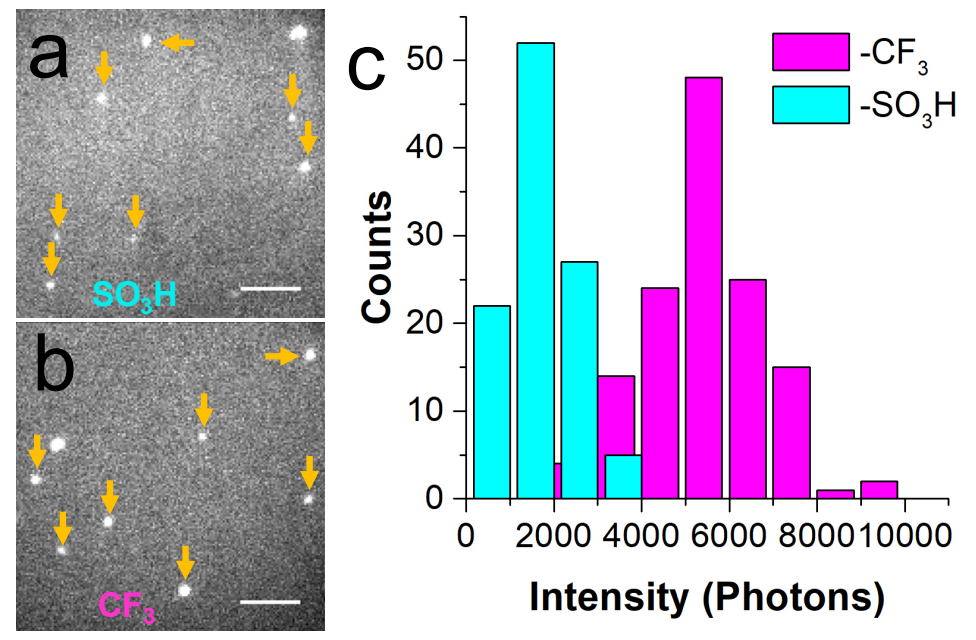

Figure S18. Enrichment of resorufin molecules in nanopores. Typical fluorescence images of single nanocatalysts of hydrophilic $\left(-\mathrm{SO}_{3} \mathrm{H}\right.$, a) and hydrophobic $\left(-\mathrm{CF}_{3}\right.$, b) nanopores. (c) Histogram analysis of fluorescence intensity in photons on single nanocatalysts $(n>100)$ of both hydrophilic and hydrophobic nanopores.

nanocatalysts of hydrophobic nanocatalysts (Figure S18c, magenta) when compared to that of hydrophilic nanopores (Figure S18c, cyan). The result suggests that resorufin molecules are more preferably enriched in hydrophobic nanopores, which can be expected since resorufin is hydrophobic. ${ }^{27}$ Amplex red molecule and Amplex red radicals are hydrophobic, like resorufin molecule, thus should also be more preferably enriched in the hydrophobic nanopores. 


\section{Supporting References}

1. Dong, B.; Pei, Y.; Mansour, N.; Lu, X.; Yang, K.; Huang, W.; Fang, N. Deciphering nanoconfinement effects on molecular orientation and reaction intermediate by single molecule imaging. Nat. Commun. 2019, 10 (1), 4815.

2. Shylesh, S.; Sharma, S.; Mirajkar, S. P.; Singh, A. P. Silica functionalised sulphonic acid groups: synthesis, characterization and catalytic activity in acetalization and acetylation reactions. J. Mol. Catal. A: Chem. 2004, 212 (1), 219.

3. Yuan, D.; Liu, Z.; Tay, S. W.; Fan, X.; Zhang, X.; He, C. An amphiphilic-like fluoroalkyl modified $\mathrm{SiO} 2$ nanoparticle@Nafion proton exchange membrane with excellent fuel cell performance. Chem. Commun. 2013, 49 (83), 9639.

4. Campos, R.; Guenthner, A. J.; Haddad, T. S.; Mabry, J. M. Fluoroalkyl-Functionalized Silica Particles: Synthesis, Characterization, and Wetting Characteristics. Langmuir 2011, 27 (16), 10206.

5. Huh, S.; Wiench, J. W.; Yoo, J.-C.; Pruski, M.; Lin, V. S. Y. Organic Functionalization and Morphology Control of Mesoporous Silicas via a Co-Condensation Synthesis Method. Chem. Mater. 2003, 15 (22), 4247.

6. Rodella, C. B.; Barrett, D. H.; Moya, S. F.; Figueroa, S. J. A.; Pimenta, M. T. B.; Curvelo, A. A. S.; Teixeira da Silva, V. Physical and chemical studies of tungsten carbide catalysts: effects of Ni promotion and sulphonated carbon. RSC Adv. 2015, 5 (30), 23874.

7. Zou, X.; Nie, X.; Tan, Z.; Shi, K.; Wang, C.; Wang, Y.; Zhao, X. Synthesis of Sulfonic AcidFunctionalized Zirconium Poly(Styrene-Phenylvinyl-Phosphonate)-Phosphate for Heterogeneous Epoxidation of Soybean Oil. Catalysts 2019, 9 (9).

8. Ravi, S.; Roshan, R.; Tharun, J.; Kathalikkattil, A. C.; Park, D. W. Sulfonic acid functionalized mesoporous SBA-15 as catalyst for styrene carbonate synthesis from $\mathrm{CO}_{2}$ and styrene oxide at moderate reaction conditions. J. of $\mathrm{CO}_{2}$ Util. 2015, 10, 88.

9. $\quad \mathrm{Xu}, \mathrm{W} . ;$ Kong, J. S.; Yeh, Y.-T. E.; Chen, P. Single-Molecule Nanocatalysis Reveals Heterogeneous Reaction Pathways and Catalytic Dynamics. Nat. Mater. 2008, 7 (12), 992.

10. Kumarasinghe, R.; Higgins, E. D.; Ito, T.; Higgins, D. A. Spectroscopic and PolarizationDependent Single-Molecule Tracking Reveal the One-Dimensional Diffusion Pathways in Surfactant-Templated Mesoporous Silica. J. Phys. Chem. C 2016, 120 (1), 715.

11. Jung, C.; Kirstein, J.; Platschek, B.; Bein, T.; Budde, M.; Frank, I.; Müllen, K.; Michaelis, J.; Bräuchle, C. Diffusion of Oriented Single Molecules with Switchable Mobility in Networks of Long Unidimensional Nanochannels. J. Am. Chem. Soc. 2008, 130 (5), 1638.

12. Feil, F.; Jung, C.; Kirstein, J.; Michaelis, J.; Li, C.; Nolde, F.; Müllen, K.; Bräuchle, C. Diffusional and Orientational Dynamics of Various Single Terylene Diimide Conjugates in Mesoporous Materials. Micropor. Mesopor. Mater. 2009, 125 (1-2), 70.

13. Higgins, D. A.; Park, S. C.; Tran-Ba, K.-H.; Ito, T. Single-Molecule Investigations of Morphology and Mass Transport Dynamics in Nanostructured Materials. Annu. Rev. Anal. Chem. 2015, 8 (1), 193.

14. Pramanik, R.; Ito, T.; Higgins, D. A. Molecular Length Dependence of Single Molecule Wobbling within Surfactant- and Solvent-Filled Silica Mesopores. J. Phys. Chem. C 2013, 117 (29), 15438.

15. Favot, F.; Corso, A. D.; Baldereschi, A. Adsorption geometry of benzene on Pd(110): Results of first-principles calculations. Europhys. Lett. 2000, 52 (6), 698.

16. Mittendorfer, F.; Hafner, J. Density-functional study of the adsorption of benzene on the (111), (100) and (110) surfaces of nickel. Surf. Sci. 2001, 472 (1), 133. 
17. Gland, J. L.; Somorjai, G. A. Low-energy electron diffraction and work function studies of adsorbed organic monolayers on the (100) and (111) crystal faces of platinum. Adv. Coll. Int. Sci. 1976, 5 (3), 205.

18. Castillejos, E.; Debouttière, P. J.; Roiban, L.; Solhy, A.; Martinez, V.; Kihn, Y.; Ersen, O.; Philippot, K.; Chaudret, B.; Serp, P. An Efficient Strategy to Drive Nanoparticles into Carbon Nanotubes and the Remarkable Effect of Confinement on Their Catalytic Performance. Angew. Chem. Int. Ed. 2009, 48 (14), 2529.

19. Jenkins, S. J. Aromatic adsorption on metals via first-principles density functional theory. Proc. $R$. Soc. A 2009, 465 (2110), 2949.

20. Dębski, D.; Smulik, R.; Zielonka, J.; Michałowski, B.; Jakubowska, M.; Dębowska, K.; Adamus, J.; Marcinek, A.; Kalyanaraman, B.; Sikora, A. Mechanism of oxidative conversion of Amplex ${ }^{\circledR}$ Red to resorufin: Pulse radiolysis and enzymatic studies. Free Radic. Biol. Med. 2016, 95, 323.

21. Gorris, H. H.; Walt, D. R. Mechanistic Aspects of Horseradish Peroxidase Elucidated through Single-Molecule Studies. J. Am. Chem. Soc. 2009, 131 (17), 6277.

22. Serra-Maia, R.; Bellier, M.; Chastka, S.; Tranhuu, K.; Subowo, A.; Rimstidt, J. D.; Usov, P. M.; Morris, A. J.; Michel, F. M. Mechanism and Kinetics of Hydrogen Peroxide Decomposition on Platinum Nanocatalysts. ACS Appl. Mater. Interfaces 2018, 10 (25), 21224.

23. Thompson, R. E.; Larson, D. R.; Webb, W. W. Precise Nanometer Localization Analysis for Individual Fluorescent Probes. Biophys. J. 2002, 82 (5), 2775.

24. Barlow, R. J. Statistics, A Guide to the Use of Statistical Methods in the Physical Sciences. John Wiley \& Sons: Chichester 1989.

25. Roeffaers, M. B. J.; Sels, B. F.; Uji-i, H.; De Schryver, F. C.; Jacobs, P. A.; De Vos, D. E.; Hofkens, J. Spatially resolved observation of crystal-face-dependent catalysis by single turnover counting. Nature 2006, 439 (7076), 572.

26. Berg, H. C. Random Walks in Biology. Princeton University Press: Princeton 1993.

27. Aumiller, W. M.; Davis, B. W.; Hashemian, N.; Maranas, C.; Armaou, A.; Keating, C. D. Coupled Enzyme Reactions Performed in Heterogeneous Reaction Media: Experiments and Modeling for Glucose Oxidase and Horseradish Peroxidase in a PEG/Citrate Aqueous Two-Phase System. $J$. Phys. Chem. B 2014, 118 (9), 2506. 